\title{
Systematic analysis of atomic protein-ligand interactions in the PDB
}

\section{Matthieu Schapira Renato Ferreira de Freitas}

Structural Genomics Consortium

We compiled a list of 11016 unique structures of small-molecule ligands bound to proteins in the Protein Databank - 6444 of which have experimental binding affinity - representing 750873 protein-ligand atomic interactions, and analyzed the frequency, geometry and impact of each interaction type. We find that hydrophobic interactions are generally enriched in high-efficiency ligands, but polar interactions are overrepresented in fragment inhibitors. While most observations extracted from the PDB will be familiar to seasoned medicinal chemists, less expected findings, such as the high number of $\mathrm{C}-\mathrm{H} \cdots \mathrm{O}$ hydrogen bonds or the relatively frequent amide- $\pi$ stacking between the backbone amide of proteins and aromatic rings of ligands, uncover underused ligand design strategies. 